\title{
Relaxation processes in Aeolian transport
}

\author{
Houssem Selmani ${ }^{1,4, \star}$, Alexandre Valance ${ }^{1, \star \star}$, Ahmed Ould El Moctar ${ }^{2}$, Pascal Dupont ${ }^{3}$, and Rabah Zegadi ${ }^{4}$ \\ ${ }^{1}$ Institut de Physique de Rennes, UMR UR1-CNRS 6251, Université de Rennes 1, 35042 Rennes cedex, France. \\ ${ }^{2}$ Laboratoire de Thermocinétique, Polytech. Nantes, CNRS UMR 6607, 44306 Nantes, France. \\ ${ }^{3}$ GCGM, INSA de Rennes, Campus Beaulieu, 35043 Rennes, France. \\ ${ }^{4}$ Laboratoire de Mécanique de Précision Appliquée, Université Ferhat Abbas Sétif 1, Algeria.
}

\begin{abstract}
.
We investigate experimentally the relaxation process toward the equilibrium regime of saltation transport in the context of spatial inhomogeneous conditions. The relaxation length associated to this process is an important length in aeolian transport. This length stands for the distance needed for the particle flux to adapt to a change in flow conditions or in the boundary conditions at the bed. Predicting the value of this length under given conditions of transport remains an open and important issue. We conducted wind tunnel experiments to document the influence of the upstream particle flux and wind speed on the relaxation process toward the saturated transport state. In the absence of upstream particle flux, data show that the relaxation length is independent of the wind strength (except close to the threshold of transport). In contrast, in the case of a finite upstream flux, the relaxation length exhibits a clear increase with increasing air flow velocity. Moreover, in the latter the relaxation is clearly non-monotonic and presents an overshoot.
\end{abstract}

\section{Introduction}

Saltation is known as the primary mode of sediment transport in air and characterizes the movement of the particles jumping along the sand surface in ballistic trajectories.[14]. When a steady wind blows over a flat erodible bed, an equilibrium between the flow and the transported particles is achieved and the mass flux, $Q$, reaches a saturated value $Q=Q_{\text {sat }}$. Now, if we consider an unsteady or inhomogeneous situation, in which the wind velocity is subjected to a temporal or spatial change, the relaxation process towards the new saturated state takes a certain characteristic time or length $[5,6]$. By linearizing the problem around the saturated state, one can describe the relaxation process by a first order differential which takes the following form for spatial inhomogeneous situations:

$$
\frac{\partial Q}{\partial x}=-\frac{Q-Q_{s a t}}{L_{s a t}}
$$

where $L_{s a t}$ is the characteristic length scale of the relaxation process and $Q_{\text {sat }}$ is the saturated state corresponding to the new equilibrium transport regime. $L_{s a t}$ is usually referred to as the saturation length $\left(L_{s a t}\right)$ [5-8]. A similar first order differential equation can be written for unsteady situations where the space variable is replaced by the time and the saturation length by the saturation time $\tau_{\text {sat }}$.

The relaxation process is driven by several intricate physical processes. If the wind speed is suddenly increased, the saltating particles will be accelerated but within a certain time delay due to particle inertia. New grains will be extracted from the bed either by aerodynamic or impact entrainment but at a certain rate depending on the efficiency of the particle pick-up process. Then, in response to the increase of the concentration of the saltating particles, the wind speed within the transport layer is lowered due to momentum extraction by the particles until a new equilibrium is reached. It turns out that several time or spatial scales may be a good candidate for the saturation time or length, respectively: the time needed for the negative feedback of transport on the wind to take place [9], the time needed to expel new grains from the sand bed [5], and the time needed to accelerate the grains to their new equilibrium velocity. It is generally argued that the latter time scale, usually referred to as the drag time $\tau_{\text {drag }}$ (or the drag length $l_{\text {drag }}$ for the corresponding length scale) [10] is associated with the slowest of these processes and is therefore expected to control the relaxation process. The drag length is the distance for a particle initially at rest to equilibrate its velocity with that of the fluid. It is controlled by the particle inertia and is given by $l_{d r a g}=\left(\rho_{p} / \rho_{a}\right) d$. Experimental estimations of the saturation length by [6] indicate that the saturation length is independent of the wind strength and is about twice the drag length: $L_{s a t}=2 l_{d r a g}$ (for $d=200 \mu \mathrm{m}$, the saturation length is on the order of a meter). Eq. (1) tells us that the mass flux relaxes monotonously towards the equilibrium saturated state via a simple exponential behavior:

$$
Q(x)=Q_{s a t}+\left(Q_{0}-Q_{s a t}\right) \exp \left(-\frac{x-x_{0}}{L_{s a t}}\right)
$$

\footnotetext{
^e-mail: selmani.h@univ-setif.dz

$\star \star$ e-mail: alexandre.valance@univ-rennes1.fr
} 
Table 1: Values of the fitted parameters obtained from the data of Andreotti et al. [6].

\begin{tabular}{cccc}
\hline$u^{\star}(\mathrm{m} / \mathrm{s})$ & $Q_{\text {sat }}\left(\mathrm{mm}^{2} / \mathrm{s}\right)$ & $x_{0}(\mathrm{~m})$ & $L_{\text {sat }}(\mathrm{m})$ \\
\hline 0.26 & 12.3555 & 0.5721 & 0.4607 \\
0.33 & 21.0209 & 0.3151 & 0.3932 \\
0.48 & 41.5917 & 0.1192 & 0.5160 \\
\hline
\end{tabular}

where $Q_{0}$ is the upstream mass flux at the position $x=x_{0}$. This simple exponential behavior is expected to provide a good description of the relaxation process as long as the upstream mass flux $Q_{0}$ is not too far from the equilibrium saturated value $Q_{\text {sat }}$. Andreotti et al. [6] reported windtunnel experiments concerning the variation of the mass flux along the downstream direction and showed the last stage of the relaxation (i.e., for $Q>Q_{\text {sat }} / 4$ ) is well described by Eq. (2) (see Fig. 1). The first stage of the relaxation process (i.e., for $0<Q<Q_{\text {sat }} / 4$ ) is however not captured by Eq. (2).

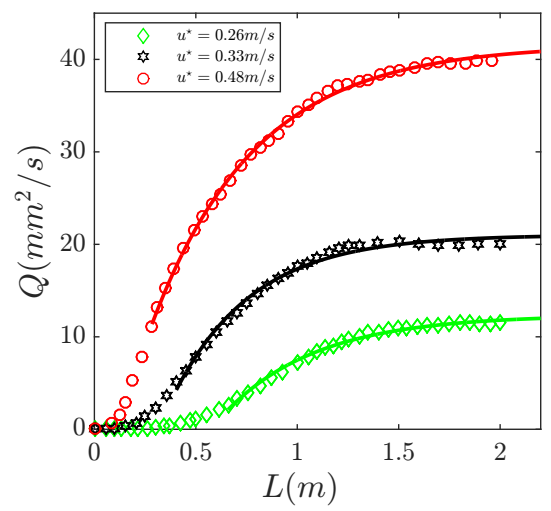

Figure 1: Variation of the mass flux along the downstream distance $x$ for three different wind friction velocities: $u^{\star}=0.26,0.33$ and $0.48 \mathrm{~m} / \mathrm{s}$ (Wind-tunnel data from Andreotti et al [6]). The upstream mass flux for the three runs is $Q_{0}=0$. The solid lines represent the best fit using eq. (2) starting from $x=x_{0}$ where $x_{0}$ is defined such $Q=Q_{\text {sat }} / 4$. See table 1 for the values of the fitted parameters $x_{0}, L_{\text {sat }}$ and $Q_{\text {sat }}$.

Other experimental results indicate more complex relaxation processes with an overshoot which is accompanied by an oscillating behavior. In particular, [1] reported wind-tunnel experiments showing that this type of complex relaxation occurs in the case where the upstream mass flux $Q_{0}$ is non-zero and controlled independently from the wind speed with a hopper device (see Fig. 2). Eq. (2) is again too simple to capture the experimental data from Bagnold.

The above experimental results illustrate the complexity of the relaxation process and its sensitivity to the outof-equilibrium situation which is investigated. The prediction and description of the relaxation processes and their characteristic length scale is an important issue in the context of the formation and dynamics of aeolian sand dunes.

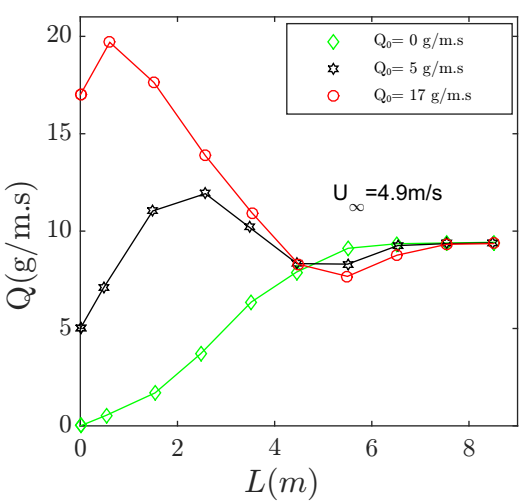

Figure 2: Variation of the mass flux along the downstream distance $x$ (Data from Bagnold [1]) for three different upstream flux conditions: $Q_{0}=0,5$ and $17 \mathrm{~g} / \mathrm{m} . \mathrm{s}$. The nominal velocity of the air flow is $U_{\infty}=4.9 \mathrm{~m} / \mathrm{s}$ for three runs.

In particular, the saturation length controls the length of the smallest dunes emerging from a flat sand bed [11, 12] and is therefore a crucial parameter in morpho-dynamic models of Earth's sandy landscapes [13-15].

Our aim here is to investigate through wind-tunnel experiments the relaxation process of the mass flux $Q$ towards its saturated value $Q_{\text {sat }}$ for different out-ofequilibrium situations. Inspired by Bagnold experiments, we conducted a series of wind-tunnel experiments varying systematically the upstream flux and wind conditions in order to document the relaxation process.

The paper is organized as follows. First, the wind tunnel facilities and protocols are described in Section 2, while results and analyses of the relaxation process are presented in Section 3. Finally, section 4 provide conclusion and outlook.

\section{Experiments}

The experiments reported here were performed in a $6.6 \mathrm{~m}$ long wind tunnel with a cross-section of dimensions $0.27 m \times 0.27 m$ where the nominal air velocity $U_{\infty}$ (i.e., the air speed outside from the boundary layer) can be varied between 0 to $20 \mathrm{~m} / \mathrm{s}$ (see [16, 17] for further details). The floor of the tunnel is covered with a sand layer whose stream-wise length can be varied from 0.2 to $6 \mathrm{~m}$. At the upstream edge of the sand bed, a hopper is installed on the roof of the wind-tunnel and allows to deliver a constant and prescribed upstream sand flux $Q_{0}$ (see Fig. 3). We used natural sand with a median diameter $d=200 \mu \mathrm{m}$ and density $\rho_{p}=2650 \mathrm{~kg} / \mathrm{m}^{3}$. The air speed is measured by using a Pitot tube located at a distance of $6 \mathrm{~m}$ from the beginning of the tunnel and set at a height of $130 \mathrm{~mm}$ from the floor. The Pitot tube is outside from the boundary layer and is used to assess the nominal air velocity $U_{\infty}$.

We performed experiments with increasing bed length $L$ from 0.2 to $6 \mathrm{~m}$ (with an increment of $0.5 \mathrm{~m}$ ) for various air speeds (from 6 to $10 \mathrm{~m} / \mathrm{s}$ ) and three different upstream sand fluxes $Q_{0}\left(Q_{0}=0,35.6\right.$ and $69.5(\mathrm{~g} / \mathrm{m} . \mathrm{s})$; cf 


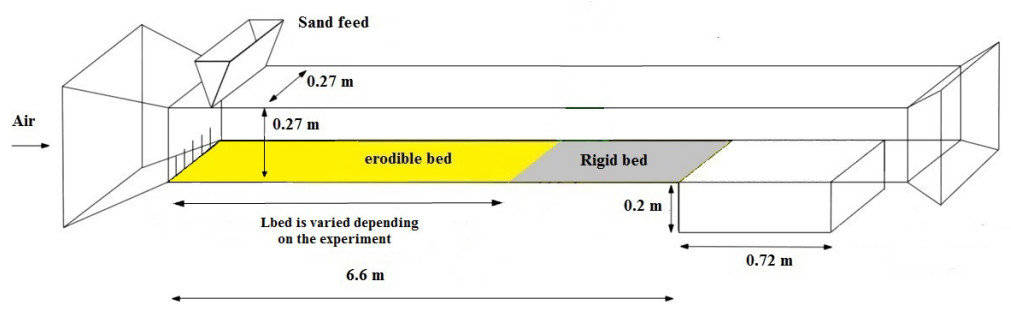

Figure 3: Schematic view of the wind tunnel.

Table 2: Air speeds $U_{\infty}$ and upstream fluxes $Q_{0}$ used in the reported experiments.

\begin{tabular}{cccc}
\hline$Q_{0}(\mathrm{~g} / \mathrm{m} . \mathrm{s})$ & 0 & 35.6 & 69.5 \\
\hline & 6.09 & 6.06 & 6.05 \\
& 7.06 & 7.05 & 7.07 \\
$U_{\infty}(\mathrm{m} / \mathrm{s})$ & 8.05 & 8.05 & 8.05 \\
& 9.07 & 9.04 & 9.05 \\
& 10.04 & 10.04 & 10.06 \\
\hline
\end{tabular}

Table 2 for further details). The non-zero values of upstream fluxes correspond to saturated values of saltation transport obtained at $U_{\infty}=7.53$ and $9.23 \mathrm{~m} / \mathrm{s}$, respectively.

Each run follows the same protocol. The sand bed is prepared with a uniform thickness of $2 \mathrm{~cm}$ and a given stream-wise bed length $L$. The sand bed surface is flattened before each run. The fan of the tunnel is tuned at a given wind speed while the hopper is opened at the same time delivering a prescribed upstream flux. A run lasts a few minutes (typically between 1 and 20 minutes according to the wind strength) in order to collect a measurable amount of sand at the exit of the tunnel. After each run, the collected sand is weighed and the resulting outgoing flux $Q(L)$ is determined as follows: $Q(L)=M / T \times W$, where $M$ is the mass of the collected sand, $T$ the duration of the run and $W$ the wind-tunnel width.

These experiments allowed to document the relaxation process and estimate the characteristic relaxation length needed to reach the saturated state of transport starting from different out-of-equilibrium situations.

\section{Results and discussion}

The evolution of the mass flux with respect to the bed length for different upstream mass fluxes and air velocities is presented in Fig. 4. In the case of a zero upstream flux, the downstream mass flux increases monotonously with increasing bed length until an asymptotic value corresponding to the equilibrium saturated value is reached. The characteristic relaxation length of the whole process decreases within increasing wind speed but saturates to a constant value of between $1 \mathrm{~m}$ and $2 \mathrm{~m}$ for nominal air velocities greater than $8 \mathrm{~m} / \mathrm{s}$. In the case of a non-zero upstream flux, the evolution of mass flux with the bed length exhibits a peak value that is greater than the equilibrium saturated flux $Q_{\text {sat }}$. This overshoot effect is caused by the process of impact entrainment triggered by the particles launched upstream. The peak value of the mass flux $Q$ increases with increasing both the upstream mass flux and air flow velocity. These data confirm the trends observed in Bagnold experiments [1]. However, unlike Bagnold observations, we do not see a clear oscillating behavior during the relaxation towards the saturated value.

Our data provide additional information about the characteristic relaxation length in case of non-zero upstream flux. Indeed, the latter increases with increasing wind strength. The influence of the upstream mass flux on the relaxation length is however not evidenced. Additional experiments would be needed to clarify this issue.

\section{Conclusion}

We carried out wind tunnel experiments to document the relaxation process towards the equilibrium regime of aeolian sand transport in the context of spatial inhomogeneous configuration by varying the upstream particle flux and wind speed. For zero upstream mass flux, the relaxation length characterizing the whole relaxation process decreases with increasing wind speed but reach an asymptotic value at large wind speed. These results confirm the measurements of Andreotti et al. showing the relaxation length (including the amplification process and the saturation one) diverge at the transport threshold. In contrast, for non-zero upstream mass flux, the relaxation length shows a clear increase with the wind strength. In addition, the relaxation exhibits, in this case, an overshoot already mentioned by Bagnold [1].

In the light of these results, it becomes clear that the simple relaxation equation (cf Eq. 1) is not able to capture the amplification process (including the overshoot) and the saturation process. It is therefore needed to model in details all the physical mechanisms involved in the relaxation process. Continuum models for aeolian sand transport have already been developed in the context of unsteady or spatial inhomogeneous conditions such as the one proposed by Sauermann et al. [5]. The next step is to make quantitative comparison with the predictions of these models and check their pertinence and validity. 

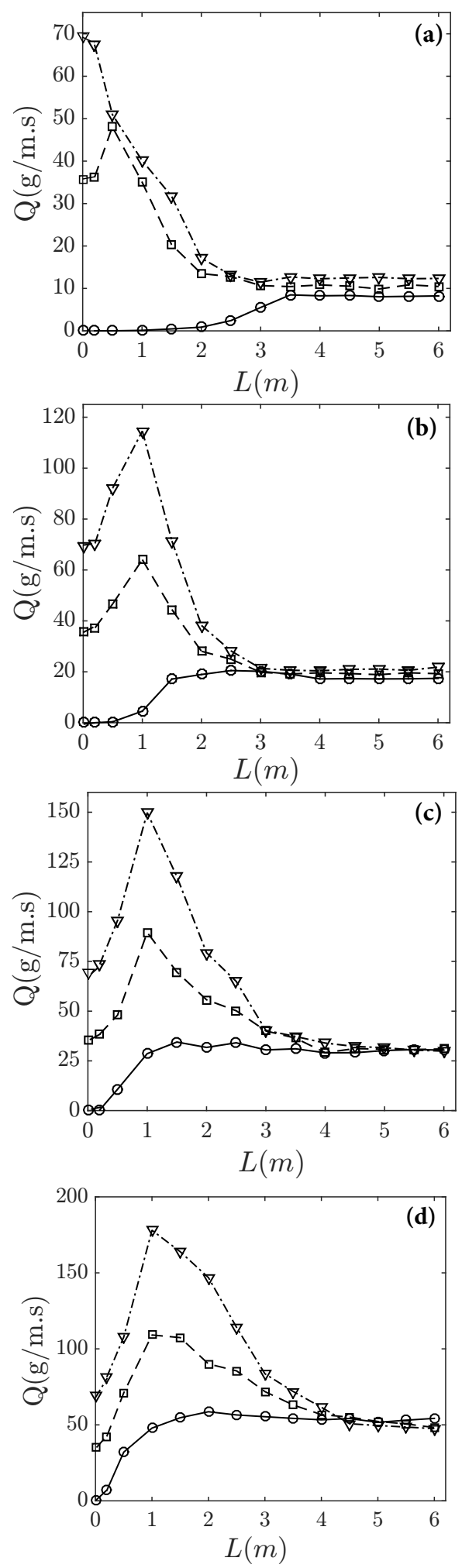

Figure 4: (a-d) The evolution of the sand mass flux as a function of the bed length $L$ for 4 different air velocities respectively $U_{\infty}=6,7,8$ and $9 \mathrm{~m} / \mathrm{s}$. For each air speed, three upstream fluxes were investigated $Q_{0}=$ 0, 35.6, $69.5 \mathrm{~g} / \mathrm{m} . \mathrm{s}$ represented respectively by (o), ( $\square$ ), $(\nabla)$.

\section{Acknowledgments}

The authors would like to thank the Algerian Ministry of Higher Education and Scientific Research for the financial support in the framework of program PNE 2015-2016.

\section{References}

[1] R.A. Bagnold, Methuen, New York (1941)

[2] O. Durán, P. Claudin, B. Andreotti, Aoelian Research 3, 243 (2011)

[3] J.F. Kok, E. Partel, T. Michael, D. Bou Karam, Report on progress in Physics 75, 106901 (2012)

[4] A. Valance, K.R. Rasmussen, A. Ould El Moctar, P. Dupont, Comptes Rendus Physique 16, 105 (2015)

[5] G. Sauermann, K. Kroy, H.J. Herrmann, Physical Review E. 64, 031305 (2001)

[6] B. Andreotti, P. Claudin, O. Pouliquen, Geomorphology 123, 343 (2010)

[7] T. Pähtz, J.F. Kok, E.J.R. Parteli, H.J. Herrmann, Physical Review Letters 111, 218002 (2013)

[8] T. Pähtz, A. Omeradžić, M.V. Carneiro, N.A.M. Araújo, H.J. Herrmann, Geophysical Research Letters 42, 2063 (2015)

[9] B. Andreotti, Journal of Fluid Mechanics 510, 47 (2004)

[10] P. Hersen, S. Douady, B. Andreotti, Physical Review Letters 89, 264 (2002)

[11] P. Claudin, B. Andreotti, Earth and Planetary Science Letters 252, 30 (2006)

[12] E.J.R. Parteli, O. Durán, H.J. Hermann, Physical Review E 75, 011301 (2007)

[13] C. Narteau, D. Zhang, O. Rozier, P. Claudin, Journal of Geophysical Research: Earth Surface 114, F03006 (2009)

[14] E.J.R. Parteli, O. Durán, H. Toar, V. Schwammle, H.J. Hermann, Proceedings of the National Academy of Science 106, 22085 (2009)

[15] E.J.R. Parteli, K. Kroy, H. Tsoar, J.S. Andrade, T. Poschel, Review and future plans, The European Physical Journal Special Topics 223, 2269 (2014)

[16] T.D. Ho, A. Valance, P. Dupont, A. Ould El Moctar, Physical Review Letters 106, 094501 (2011)

[17] T.D. Ho, A. Valance, P. Dupont, A. Ould El Moctar, Aeolian Research 223, pp. 65-74 (2014) 